\title{
Variable Control Charts Based on Percentiles of the New Weibull-Pareto Distribution
}

\author{
Srinivas Rao Boyapati \\ Department of Mathematics \& Humanities \\ R.V.R \& J.C College of Engineering \\ Chowdavaram, Guntur- 522019 \\ Andhra Pradesh, India \\ boyapatisrinu@yahoo.com \\ Suleman Nasiru \\ Department of Statistics, Faculty of Mathematical Sciences \\ University for Development Studies, P.O.Box24, Navrongo \\ Upper East Region, Ghana, West Africa. Ghana \\ sulemanstat@gmail.com \\ K.N.V.R. Lakshmi \\ Department of Statistics, Acharya Nagarjuna University \\ Guntur- 522 010, Andhra Pradesh, India \\ knvrlakshmi@gmail.com
}

\begin{abstract}
A variable quality characteristic is assumed to follow the new Weibull- Pareto distribution. Based on the evaluated percentiles of sample statistics like mean, median, midrange, range and standard deviation, the control limits for the respective control charts are developed. The admissibility and power of the control limits are assessed in comparison with those based on the popular Shewhart control limits.
\end{abstract}

Keywords and phrases: Most probable, Pdf, Cdf, Equi-tailed, Percentiles, NWPD.

\section{Introduction}

The well-known Shewhart control charts are developed under the assumption that the quality characteristic follows a normal distribution. If $x_{1}, x_{2}, \ldots x_{\mathrm{n}}$ is a collection of observations of size $n$ on a variable quality characteristic of a product and if $t_{\mathrm{n}}$ is a statistic based on this sample, the control limits of Shewhart variable control chart are $\mathrm{E}\left(\mathrm{t}_{\mathrm{n}}\right) \pm 3 S$.E $\left(\mathrm{t}_{\mathrm{n}}\right)$. In quality control studies data is always in small samples only. Therefore if the population is not normal there is a need to develop separate procedure for the construction of control limits. In this paper we assume that the quality variate follows the new Weibull-Pareto model and develop control limits for such data on par with the presently available control limits. If a process quality characteristic is assumed to follow the new Weibull-Pareto distribution the online process of such a quality can be controlled through the theory of the new Weibull-Pareto distribution. In the absence of any such specification of the population model we generally use the normal distribution and the associated constants available in all standard text books of statistical quality control. However, normality is only an assumption that is rarely verified and found to be true. Unless the sample is very large in size this assumption may not be taken for granted without proper goodness of fit test procedure. At the same time central limit theorem cannot be made use of, because central limit theorem gives only asymptotic normality for 
any statistic. Therefore, if a distribution other than normal is a suitable model for a quality variate, separate procedures are to be developed. We present the construction of quality control charts when the process variate is assumed to follow the new WeibullPareto distribution. Let $\mathrm{X}$ be a random variable from a Pareto distribution with its cumulative distribution function (cdf) for $x \geq \theta$ given by

$$
F_{1}(x ; \theta, k)=1-\left(\frac{\theta}{x}\right)^{k}
$$

where $\theta>0$ is a scale parameter and $\mathrm{k}>0$ is the shape parameter.

The probability density function (pdf) corresponding to (1.1) is

$$
f_{1}(x ; \theta, k)=\frac{k \theta^{k}}{x^{k+1}}
$$

The new Weibull-Pareto distribution has a cdf of the form

$$
F(x)=\int_{0}^{\frac{1}{R(x)}} f_{2}(x) d x
$$

where $\mathrm{R}(x)$ is the survival function of the Pareto distribution and is given by

$\mathrm{R}(x)=1-F_{1}(x ; \theta, k)$ while $f_{2}(x)$ is the pdf of a Weibull distribution and is given by

$$
f_{2}(x)=\alpha \lambda(\lambda x)^{\alpha-1} e^{-(\lambda x) \alpha}
$$

where $\mathrm{x}>0, \alpha>0$ and $\lambda>0$.

Using (1.3) and (1.4) given that $\mathrm{R}(x)=\left(\frac{\theta}{x}\right)^{k}$, the cdf of the NWPD is given by

$$
\begin{aligned}
& F(x)=\int_{0 x}^{\frac{1}{\left(-\frac{\theta}{k}\right.}} \alpha \lambda(\lambda x)^{\alpha-1} e^{-(\lambda x) \alpha} d x \\
& F(x)=1-e^{-\lambda \theta}\left(\frac{x}{\theta}\right)^{\alpha \kappa}
\end{aligned}
$$

If we let $\delta=\lambda^{\alpha}$ and $\beta=\alpha \kappa$, then the cdf of the new Weibull-Pareto distribution (NWPD) can be written as

$$
F(x)=1-e^{-\delta\left(\frac{x}{\theta}\right) \beta}
$$

The pdf is given by

$$
g(x)=\frac{\beta \delta}{\theta}\left(\frac{x}{\theta}\right)^{\beta-1} e^{-\delta\left(\frac{x}{\theta}\right) \beta}
$$

where $0<x<\infty, \beta>0, \theta>0$ and $\delta>0$.

The hazard function is given by

$$
h(x)=\frac{\beta \delta}{\theta}\left(\frac{x}{\theta}\right)^{\beta-1}
$$


From the hazard function the following can be observed:

(1) if $\beta=1$, the failure rate is constant, which makes the NWPD suitable for modeling systems or components with failure rate.

(2) if $\beta>1$, the hazard is an increasing function, which makes the NWPD suitable for modeling components that wears faster with time.

(3) if $\beta<1$, the hazard is a decreasing function, which makes the NWPD suitable for modeling components that wears slower with time.

The distributional properties are:

$$
\begin{aligned}
& E(x)=\theta \delta^{\frac{-1}{\beta}} \Gamma\left(\frac{\beta+1}{\beta}\right) \\
& \text { median }=\theta\left(\frac{1}{\delta} \operatorname{in}(2)\right)^{\frac{1}{\beta}} \\
& \text { Variance }=\quad \theta 2 \delta^{\frac{-2}{\beta}} \Gamma\left(\frac{\beta+2}{\beta}\right)-\left[\theta \delta^{\frac{-1}{\beta}} \Gamma\left(\frac{\beta+1}{\beta}\right)\right]^{2}
\end{aligned}
$$

The pdf the largest order statistic $X_{(\mathrm{n})}$ is given by

$$
\alpha_{(n)}=\frac{n \beta \delta}{\theta}\left(\frac{x}{\theta}\right)^{n-1} e^{-\delta\left(\frac{x}{\theta}\right)} \beta=\left[1-e^{-\delta\left(\frac{x}{\theta}\right)} \beta\right]^{n-1}
$$

The pdf of the smallest order statistic $X_{(1)}$ is given by

$$
\alpha_{(1)}=\frac{n \beta \delta}{\theta}\left(\frac{x}{\theta}\right)^{n-1} e^{-\delta\left(\frac{x}{\theta}\right)} \times\left[e^{-\delta\left(\frac{x}{\theta}\right)^{\beta}}\right]^{n-1}
$$

The other distributional properties are thoroughly discussed by Nasiru and Luguterah (2015) [8]. Skewed distributions to develop statistical quality control methods are attempted by many authors. Some of them are Edge-man(1989) [3] - Inverse Gaussion Distribution, Gonzalez and Viles (2000) [4] - Gamma Distribution, Kantam and Sriram(2011) [5]-Gamma Distribution, Chan and Cui (2003) [2] have developed control chart constants for skewed distributions where the constants are dependent on the coefficient of skewness of the distribution, Kantam et al (2006) [6] - Log logistic Distribution, Betul and Yaziki (2006) [1]- Burr Distribution, Subba Rao and Kantam(2008) [11]-Double exponential distribution, Kantam and Rao(2010) [7]-control charts for process variate, Rao and Sarath Babu (2012) [9]-Linear failure rate distribution, Rao and Kantam (2012) [10]-Half logistic distribution and references there in.

NWPD is another situation of skewed distribution which is paid much attention with respect to development of control charts in the present study. If $\beta=0.5, \delta 1.5$ and $\theta=2.0$, then the hazard function indicates a decreasing failure rate function (shown in the graph), which makes the NWPD suitable for modeling components that wears slower with time. At the same time it is one of the probability models applicable for life testing and reliability studies also. Accordingly, if a lifetime data is considered as a quality data, 
development of control charts for the same is desirable for the use by practitioners. Since NWPD is a skewed distribution, this paper makes an attempt to study in a comparative manner. An attempt is made in this paper to address this problem and solve it to the extent possible. The rest of the paper is organized as follows. The basic theory and the development of control charts for the statistics - mean, median, midrange, range and standard deviation are presented in Section 2. The comparative study to the developed control limits in relation to the Shewhart limits is given in Section 3. Summary and conclusions are given in Section 4.

Figure 1: Hazard function of the new Weibull-Pareto distribution

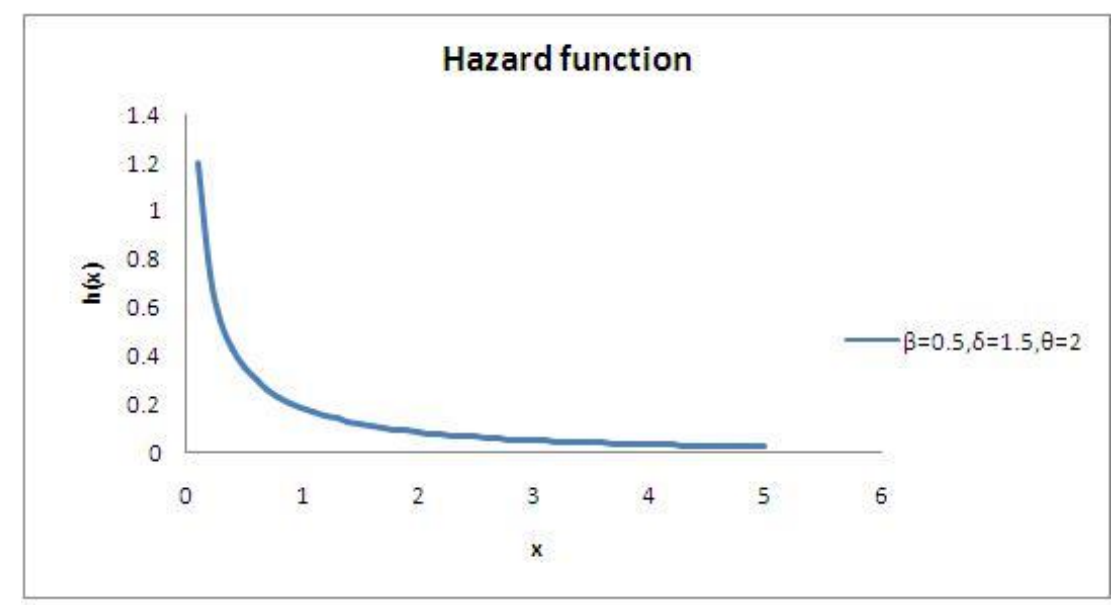

\section{Control chart constants through percentiles}

\subsection{Mean-chart.}

Let $x_{1}, x_{2}, . ., x_{\mathrm{n}}$ be a random sample of size $\mathrm{n}$ supposed to have been drawn from NWPD with $\beta=0.5, \delta=1.5$ and $\theta=2.0$. If this is considered as a subgroup of an industrial process data with a targeted population average, under repeated sampling the statistic $\bar{x}$ gives whether the process average is around the targeted mean or not. Statistically speaking, we have to find the 'most probable' limits within which $\bar{x}$ falls. Here the phrase 'most probable' is a relative concept which is to be considered in the population sense. As the existing procedures are for normal distribution only, the concept of $3 \sigma$ limits is taken as the 'most probable' limits. It is well known that $3 \sigma$ limits of normal distribution include 99.73\% of probability. Hence, we have to search two limits of the sampling distribution of sample mean in NWPD such that the probability content of those limits is 0.9973 . Symbolically we have to find L, U such that

$$
P(L \leq \bar{x} \leq U)=0.9973
$$

Where $\bar{x}$ is the mean of the sample size $n$. Taking the equi-tailed concept $\mathrm{L}, \mathrm{U}$ are respectively 0.00135 and 0.99865 percentiles of the sampling distribution of $\bar{x}$. We resorted to the empirical sampling distribution of $\bar{x}$ through simulation there by computing its percentiles. These are given in Table 1. 
Table 1: Percentiles of Mean in NWPD

\begin{tabular}{|l|l|l|l|l|l|l|l|l|l|l|}
\hline $\mathrm{n}$ & 0.99865 & 0.9950 & 0.99 & 0.975 & 0.95 & 0.05 & 0.025 & 0.01 & 0.005 & 0.00135 \\
\hline 2 & 17.4606 & 10.8759 & 8.0867 & 5.1371 & 3.0376 & 0.0235 & 0.0186 & 0.0151 & 0.0133 & 0.0117 \\
3 & 15.1037 & 8.7693 & 6.6472 & 4.4846 & 2.9126 & 0.0318 & 0.0257 & 0.0203 & 0.0174 & 0.0147 \\
4 & 11.7977 & 7.3706 & 5.7197 & 3.9353 & 2.7897 & 0.0385 & 0.0323 & 0.0255 & 0.0218 & 0.0182 \\
5 & 9.7210 & 6.4674 & 5.3696 & 3.6817 & 2.6375 & 0.0434 & 0.0369 & 0.0308 & 0.0273 & 0.0221 \\
6 & 8.2124 & 5.8684 & 4.6866 & 3.3315 & 2.4230 & 0.0480 & 0.0408 & 0.0344 & 0.0302 & 0.0250 \\
7 & 7.6891 & 5.7796 & 4.3089 & 3.1712 & 2.3289 & 0.0507 & 0.0441 & 0.0377 & 0.0337 & 0.0277 \\
8 & 7.1562 & 5.3255 & 3.9993 & 2.9262 & 2.2647 & 0.0541 & 0.0467 & 0.0402 & 0.0362 & 0.0299 \\
9 & 6.6775 & 4.7624 & 3.7982 & 2.7964 & 2.1887 & 0.0578 & 0.0501 & 0.0433 & 0.0389 & 0.0333 \\
10 & 6.1537 & 4.6119 & 3.6615 & 2.7455 & 2.1279 & 0.0618 & 0.0537 & 0.0460 & 0.0410 & 0.0350 \\
\hline
\end{tabular}

The percentiles in the above table are used in the following manner to get the control limits for sample mean. From the distribution of $\bar{x}$, consider

$$
\mathrm{P}\left(\mathrm{Z}_{0.00135} \leq \bar{x} \leq \mathrm{Z}_{0.99865}\right)=0.9973
$$

But $\bar{x}$ of sampling distribution when $\beta=0.5, \delta=1.5$ and $\theta=2.0$ is 1.7777 for NWPD. From equation(2.2) over repeated sampling, for the $i^{\text {th }}$ subgroup mean we can have

$$
P\left(Z_{0.00135} \frac{\bar{x}}{1.777} \leq \bar{x}_{i} \leq Z_{0.99865} \frac{=\bar{x}}{1.777}\right)=0.9973
$$

This can be written as

$$
P\left(A_{2 p}^{*} \times \bar{x} \leq \bar{x}_{i} \leq A_{2 p}^{* *} \bar{x}\right)=0.9973
$$

Where $\bar{x}$ is grand mean, $\bar{x}_{\mathrm{i}}$ is $i^{\text {th }}$ subgroup mean, $A_{2 p}^{*}=\frac{z_{0.000135}}{1.7777}, A_{2 p}^{* *}=\frac{Z_{0.99865}}{1.7777}$, Thus $A_{2 p}^{*}, A_{2 p}^{* *}$ are the percentile constants of $\bar{x}$ chart for NWPD are given in Table 2.

Table 2: Percentile constants of Mean-chart

\begin{tabular}{|l|l|l|}
\hline $\mathrm{n}$ & $A_{2 p}^{*}$ & $A_{2 p}^{* *}$ \\
\hline 2 & 9.8217 & 0.0066 \\
3 & 8.4959 & 0.0083 \\
4 & 6.6362 & 0.0102 \\
5 & 5.4681 & 0.0124 \\
6 & 4.6195 & 0.0141 \\
7 & 4.3251 & 0.0156 \\
8 & 4.0254 & 0.0168 \\
9 & 3.7561 & 0.0188 \\
10 & 3.4615 & 0.0197 \\
\hline
\end{tabular}




\subsection{Median-chart}

We have to search two limits of the sampling distribution of sample median in NWPD such that the probability content of these limits is 0.9973 . Symbolically, we have to find L, U such that

$$
\mathrm{P}(L \leq m \leq U)=0.9973
$$

Where $\mathrm{m}$ is the median of sample size $\mathrm{n}$. Through simulation, the percentiles observed are given in Table 3.

Table 3: Percentiles of Median in NWPD

\begin{tabular}{|l|l|l|l|l|l|l|l|l|l|l|}
\hline $\mathrm{n}$ & 0.99865 & 0.9950 & 0.99 & 0.975 & 0.95 & 0.05 & 0.025 & 0.01 & 0.005 & 0.00135 \\
\hline 2 & 17.4606 & 10.8759 & 8.0867 & 5.1371 & 3.0376 & 0.0235 & 0.0186 & 0.0151 & 0.0133 & 0.0117 \\
3 & 5.9335 & 4.0277 & 2.6446 & 1.4857 & 0.7665 & 0.0203 & 0.0164 & 0.0140 & 0.0127 & 0.0114 \\
4 & 4.5757 & 2.8170 & 2.1675 & 1.3434 & 0.7944 & 0.0277 & 0.0223 & 0.0184 & 0.0162 & 0.0141 \\
5 & 3.1791 & 1.7332 & 1.2423 & 0.6366 & 0.2653 & 0.0255 & 0.0212 & 0.0173 & 0.0155 & 0.135 \\
6 & 2.0708 & 1.3295 & 0.9849 & 0.6082 & 0.3367 & 0.0311 & 0.0261 & 0.0218 & 0.0191 & 0.0154 \\
7 & 1.5430 & 1.0200 & 0.7286 & 0.3027 & 0.1399 & 0.0290 & 0.0243 & 0.0203 & 0.0180 & 0.0157 \\
8 & 1.3092 & 0.8493 & 0.6391 & 0.3668 & 0.1927 & 0.0333 & 0.0284 & 0.0240 & 0.0208 & 0.0173 \\
9 & 1.0695 & 0.6782 & 0.4413 & 0.1980 & 0.1336 & 0.0322 & 0.0274 & 0.0234 & 0.0207 & 0.0179 \\
10 & 1.0399 & 0.5928 & 0.4215 & 0.2016 & 0.1340 & 0.0351 & 0.0306 & 0.0262 & 0.0235 & 0.0201 \\
\hline
\end{tabular}

The percentiles in the above table are used in the following manner to get the control limits for median. From the distribution of $\mathrm{m}$, consider

$$
P\left(Z_{0.00135} \leq m \leq Z_{0.99865}\right)=0.9973
$$

But median of sampling distribution when $\beta=0.5, \delta=1.5$, and $\theta=2.0$ is 0.42707 for NWPD.

From equation (2.6) over repeated sampling, for the $i^{\text {th }}$ subgroup median we can have

$$
P\left(Z_{0.00135} \frac{\bar{m}}{0.42707} \leq m_{i} \leq Z_{0.99865} \frac{\bar{m}}{0.42707}\right)
$$

This can be written as

$$
P\left(A_{7 p}^{*} \bar{m} \leq \bar{m}_{i} \leq A_{7 p}^{* *} \bar{m}\right)=0.9973
$$

where $\bar{m}$ is mean of subgroup medians. Thus $A_{7 p}^{*}=\frac{Z_{0.00135}}{0.42707}, A_{7 p}^{* *}=\frac{Z_{0.99865}}{0.42707}$ are the percentile constants of median chart and are given in Table 4. 
Table 4: Percentile constants of Median-chart

\begin{tabular}{|l|l|l|}
\hline $\mathrm{n}$ & $A_{2 p}^{*}$ & $A_{2 p}^{* *}$ \\
\hline 2 & 40.8847 & 0.0273 \\
3 & 13.8935 & 0.0268 \\
4 & 10.7141 & 0.0329 \\
5 & 7.4439 & 0.0317 \\
6 & 4.8487 & 0.0362 \\
7 & 3.6130 & 0.0367 \\
8 & 3.0656 & 0.0404 \\
9 & 2.5044 & 0.0419 \\
10 & 2.4349 & 0.0470 \\
\hline
\end{tabular}

\subsection{Midrange-chart.}

We have to search two limits of the sampling distribution of sample midrange in NWPD such that the probability content of these limits is 0.9973. Symbolically, we have to find $\mathrm{L}$, U such that

$$
\mathrm{P}(L \leq M \leq U)=0.9973
$$

where $\mathrm{M}$ is the midrange of sample size $\mathrm{n}$. Through simulation, the percentiles observed are given in Table 5.

Table 5: Percentiles of Midrange in NWPD

\begin{tabular}{|l|l|l|l|l|l|l|l|l|l|l|}
\hline $\mathrm{n}$ & 0.99865 & 0.9950 & 0.99 & 0.975 & 0.95 & 0.05 & 0.025 & 0.01 & 0.005 & 0.00135 \\
\hline 2 & 17.4606 & 10.8759 & 8.0867 & 5.1371 & 3.0376 & 0.0235 & 0.0186 & 0.0151 & 0.0133 & 0.0117 \\
3 & 22.6093 & 12.4842 & 9.5671 & 6.2095 & 3.9511 & 0.0327 & 0.0268 & 0.0208 & 0.0179 & 0.0184 \\
4 & 22.6993 & 13.5701 & 10.5881 & 7.0163 & 4.7670 & 0.0415 & 0.0346 & 0.0274 & 0.0232 & 0.0184 \\
5 & 23.4614 & 15.0277 & 11.6465 & 7.9251 & 5.4832 & 0.0499 & 0.0417 & 0.0343 & 0.0297 & 0.0235 \\
6 & 23.4643 & 15.1905 & 12.3891 & 8.6729 & 6.0953 & 0.0566 & 0.0485 & 0.0393 & 0.0345 & 0.0290 \\
7 & 23.4790 & 17.1724 & 12.5901 & 9.2402 & 6.6306 & 0.0609 & 0.0532 & 0.0447 & 0.0393 & 0.0320 \\
8 & 24.4671 & 18.0809 & 13.4355 & 9.5140 & 6.9891 & 0.0640 & 0.0566 & 0.0488 & 0.0433 & 0.0354 \\
9 & 25.6823 & 18.8002 & 14.0853 & 10.0124 & 7.4294 & 0.0690 & 0.0613 & 0.0532 & 0.0475 & 0.0381 \\
10 & 27.2125 & 19.6400 & 15.0062 & 10.8240 & 7.9756 & 0.0731 & 0.0659 & 0.0587 & 0.0547 & 0.0460 \\
\hline
\end{tabular}

The percentiles from the above table are used in the following manner to get the control limits for midrange. From the distribution of M, consider

$$
\mathrm{P}\left(\mathrm{z} 0.00135 \leq M \leq \mathrm{z}_{0.099865}\right)=0.9973
$$

The midrange value of NWPD is calculated by using $\alpha_{(1)}$ and $\alpha_{(\mathrm{n})}$. 
From equation (2.10) for $i^{\text {th }}$ subgroup midrange we can have,

$$
P\left(Z_{0.00135} \frac{\bar{M}}{\frac{\alpha_{(1)}+\alpha_{(n)}}{2}} \leq M_{i} \leq Z_{0.99865} \frac{\bar{M}}{\frac{\alpha(1)+\alpha(n)}{2}}\right)
$$

This can be written as

$$
P\left(A_{4 p}^{*} \bar{M} \leq M_{i} \leq A_{4 p}^{* *} \bar{M}\right)=0.9973
$$

where $\bar{M}$ is mean of midranges. Thus $A_{4 p}^{*}=\frac{2 Z_{0.0135}}{\alpha_{(1)}+\alpha_{(n)}}, A_{4 p}^{* *}=\frac{2 Z_{0.99865}}{\alpha_{(1)}+\alpha_{(n)}}$ are the percentile constants of midrange chart for NWPD process data given in Table 6.

Table 6: Percentile constants of Midrange-chart

\begin{tabular}{|l|l|l|}
\hline $\mathrm{n}$ & $A_{2 p}^{*}$ & $A_{2 p}^{* *}$ \\
\hline 2 & 831.6948 & 0.0025 \\
3 & 74.6447 & 0.0027 \\
4 & 18.9612 & 0.0028 \\
5 & 12.6372 & 0.0035 \\
6 & 8.4244 & 0.0048 \\
7 & 6.3816 & 0.0057 \\
8 & 5.4154 & 0.0059 \\
9 & 4.2019 & 0.0066 \\
10 & 2.9148 & 0.0069 \\
\hline
\end{tabular}

\subsection{R-Chart.}

We have to search two limits of the sampling distribution of sample range in NWPD such that the probability content of these limits is 0.9973 . Symbolically, we have to find $\mathrm{L}, \mathrm{U}$ such that

$$
\mathrm{P}(L \leq R \leq U)=0.9973
$$

where $\mathrm{R}$ is the range of sample of size $\mathrm{n}$. Through simulation, the percentiles observed are given in Table 7 .

Table 7: Percentiles of Range in NWPD

\begin{tabular}{|l|c|c|c|c|c|c|c|c|c|l|}
\hline $\mathrm{n}$ & 0.99865 & 0.9950 & 0.99 & 0.975 & 0.95 & 0.05 & 0.025 & 0.01 & 0.005 & 0.00135 \\
\hline 2 & 34.2650 & 20.8141 & 14.4759 & 9.0737 & 5.5478 & 0.0049 & 0.0025 & 0.0011 & 0.0006 & 0.0002 \\
3 & 45.1667 & 24.7559 & 18.9930 & 12.2815 & 7.7970 & 0.0230 & 0.0152 & 0.0095 & 0.0062 & 0.0031 \\
4 & 45.1837 & 27.0879 & 21.1320 & 13.9184 & 9.4607 & 0.0424 & 0.0313 & 0.0209 & 0.0168 & 0.0085 \\
5 & 46.8067 & 29.9866 & 23.2380 & 15.8182 & 10.8957 & 0.0608 & 0.0467 & 0.0341 & 0.0270 & 0.0180 \\
6 & 46.8871 & 30.3313 & 24.7370 & 17.3004 & 12.1545 & 0.0747 & 0.0616 & 0.0466 & 0.0387 & 0.0293 \\
7 & 46.8951 & 34.3161 & 25.1334 & 18.4278 & 13.2014 & 0.0867 & 0.0745 & 0.0580 & 0.0488 & 0.0345 \\
8 & 48.9082 & 36.1374 & 26.8441 & 18.9777 & 13.9462 & 0.0963 & 0.0801 & 0.0674 & 0.0567 & 0.0426 \\
9 & 51.3310 & 37.5642 & 28.1267 & 19.9926 & 14.8265 & 0.1066 & 0.0904 & 0.0750 & 0.0663 & 0.0494 \\
10 & 54.3942 & 39.2441 & 29.9866 & 21.6165 & 15.9104 & 0.1145 & 0.1021 & 0.0888 & 0.0787 & 0.0644 \\
\hline
\end{tabular}


The percentiles from the above table are used in the following manner to get the control limits for sample range. From distribution of $\mathrm{R}$, consider

$$
\mathrm{P}\left(\mathrm{Z}_{0.00135} \leq R \leq \mathrm{Z}_{0.99865}\right)=0.9973
$$

From equation (2.14), for the $1^{\text {th }}$ subgroup range we can have

$$
P\left(Z_{0.00135} \frac{\bar{R}}{\alpha_{(n)}-\alpha_{(1)}} \leq R_{i} \leq Z_{0.99865} \frac{\bar{R}}{\alpha_{(n)}-\alpha_{(1)}}\right)=0.9973
$$

This can be written as

$$
P\left(D_{3 p}^{*} \bar{R} \leq R_{i} \leq D_{4 p}^{* *} \bar{R}\right)=0.9973
$$

where $\bar{R}$ is mean of ranges, $\mathrm{R}_{i}$ is $i^{\text {th }}$ subgroup range. Thus $D_{3 p}^{*}=\frac{Z_{0.00135}}{\alpha_{(n)}-\alpha_{(1)}}, D_{4 p}^{* *}=\frac{Z_{0.99865}}{\alpha_{(n)}-\alpha_{(1)}}$ are the percentile constants or R chart for NWPD process data and are given in Table 8 .

Table 8: Percentile constants of Range-chart

\begin{tabular}{|l|l|l|}
\hline $\mathrm{n}$ & $A_{2 p}^{*}$ & $A_{2 p}^{* *}$ \\
\hline 2 & 980.4490 & 0.0001 \\
3 & 136.4872 & 0.0005 \\
4 & 18.4992 & 0.0009 \\
5 & 12.6301 & 0.0017 \\
6 & 8.7324 & 0.0037 \\
7 & 6.1692 & 0.0048 \\
8 & 5.2819 & 0.0052 \\
9 & 4.3546 & 0.0054 \\
10 & 3.1214 & 0.0065 \\
\hline
\end{tabular}

\section{5. $\sigma$ - chart.}

We have to search two limits of the sampling distribution of sample standard deviation in NWPD such that the probability content of these limits is 0.9973. Symbolically, we have to find $\mathrm{L}, \mathrm{U}$ such that

$$
\mathrm{P}(L \leq s \leq U)=0.9973
$$

where is the standard deviation of sample of size $\mathrm{n}$. Through simulation the percentiles observed are given in Table 9.

Table 9: Percentiles of Standard deviation in NWPD

\begin{tabular}{|l|c|c|c|c|c|c|c|c|c|c|}
\hline $\mathrm{n}$ & 0.99865 & 0.9950 & 0.99 & 0.975 & 0.95 & 0.05 & 0.025 & 0.01 & 0.005 & 0.00135 \\
\hline 2 & 17.1325 & 10.4071 & 7.2380 & 4.5368 & 2.7739 & 0.0024 & 0.0013 & 0.0005 & 0.0003 & 0.0001 \\
3 & 21.2813 & 11.5345 & 8.8545 & 5.6890 & 3.6081 & 0.0097 & 0.0066 & 0.0041 & 0.0027 & 0.0013 \\
4 & 19.5441 & 11.6939 & 8.9431 & 5.9253 & 4.0238 & 0.0165 & 0.0122 & 0.0083 & 0.0064 & 0.0033 \\
5 & 18.2745 & 11.9902 & 9.1641 & 6.3103 & 4.3426 & 0.0224 & 0.0173 & 0.0128 & 0.0097 & 0.0066 \\
6 & 17.4450 & 11.2582 & 9.0273 & 6.3984 & 4.4944 & 0.0262 & 0.0215 & 0.0163 & 0.0140 & 0.0107 \\
7 & 16.4010 & 11.8551 & 8.8169 & 6.4205 & 4.5818 & 0.0301 & 0.0254 & 0.0199 & 0.0168 & 0.0121 \\
8 & 16.0333 & 12.0707 & 8.8157 & 6.2189 & 4.6598 & 0.0323 & 0.0273 & 0.0221 & 0.0193 & 0.0137 \\
9 & 15.9565 & 11.9852 & 8.8994 & 6.2719 & 4.7164 & 0.0349 & 0.0298 & 0.0249 & 0.0221 & 0.0169 \\
10 & 16.0461 & 11.9484 & 9.0755 & 6.4586 & 4.8295 & 0.0377 & 0.0326 & 0.0282 & 0.0255 & 0.0213 \\
\hline
\end{tabular}


The percentiles from the above table are used in the following manner to get the control limits for sample standard deviation. From distribution of s, consider

$$
\mathrm{P}\left(\mathrm{Z}_{0.00135} \leq s \leq \mathrm{Z}_{0.99865}\right)=0.9973
$$

But standard deviation of sampling distribution when $\beta=0.5, \delta=1.5$ and $\theta=2.0$ is 3.9752 for NWPD. From equation (2.18), for the $1^{\text {th }}$ subgroup standard deviation we can have

$$
P\left(Z_{0.00135} \frac{\bar{S}}{3.9752} \leq S_{i} \leq Z_{0.99865} \frac{\bar{S}}{3.9752}\right)=0.9973
$$

This can be written as

$$
P\left(B_{3 p}^{*} \bar{S} \leq S_{i} \leq B_{4 p}^{* *} \bar{S}\right)=0.9973
$$

where $\bar{S}$ is mean of standard deviation, $\mathrm{s}_{i}$ is $i^{\text {th }}$ subgroup standard deviation. Thus $B_{3 p}^{*}=\frac{Z_{0.00135}}{3.9752}, B_{4 p}^{* *}=\frac{Z_{0.99865}}{3.9752}$ are the constants of standard deviation chart for NWPD process data given in Table 10.

Table 10: Percentile constants of SD-chart

\begin{tabular}{|l|l|l|}
\hline $\mathrm{n}$ & $A_{2 p}^{*}$ & $A_{2 p}^{* *}$ \\
\hline 2 & 4.3098 & 0.0001 \\
3 & 5.3535 & 0.0003 \\
4 & 4.9165 & 0.0008 \\
5 & 4.5971 & 0.0017 \\
6 & 4.3885 & 0.0027 \\
7 & 4.1258 & 0.0030 \\
8 & 4.0333 & 0.0034 \\
9 & 4.0140 & 0.0042 \\
10 & 4.0365 & 0.0054 \\
\hline
\end{tabular}

\section{Comparative study}

The control chart constants for the statistics mean, median, midrange, range and standard deviation developed in section 2 are based on the population described by NWPD. In order to use this for a data, the data is confirmed to follow NWPD. Therefore the power of the control limits can be assessed through their application for a true NWPD data in relation to the application for Shewhart limits. With this back drop we have made this comparative study simulating random samples of size $n=2, \ldots, 10$ from NWPD and calculated the control limits using the constants of section 2 for mean, median, midrange, range and standard deviation in succession. The number of statistic values that have fallen within the respective control limits is evaluated and is named as NWPD coverage probability. Similar count for control limits using Shewhart constants available in quality control manuals are also calculated. These are named as Shewhart coverage probability. The coverage probabilities under the two schemes namely true NWPD, Shewhart limits are presented in the following Tables 11, 12, 13, 14 and 15. 
Table 11: Coverage Probabilities of Mean-chart

\begin{tabular}{|l|c|l|l|l|l|c|}
\hline & \multicolumn{3}{|c|}{ Shewhart limits } & \multicolumn{3}{c|}{ Percentile limits } \\
\hline $\mathrm{n}$ & $\overline{\bar{x}}-A_{2} \bar{R}$ & $\begin{array}{l}\bar{x}+A_{2} \bar{R} \\
\text { Coverage } \\
\text { probability }\end{array}$ & $\begin{array}{c}A_{2 p}^{*} \times \bar{x} \\
A_{2 p}^{* *} \times \bar{x}\end{array}$ & $\begin{array}{c}\text { Coverage } \\
\text { probability }\end{array}$ \\
\hline 2 & 0 & 2.672798 & $\mathbf{0 . 9 4 0 2}$ & 0.004188 & 6.231820 & $\mathbf{0 . 9 8 3 1}$ \\
3 & 0 & 2.34902 & $\mathbf{0 . 9 3 2 8}$ & 0.005363 & 5.489357 & $\mathbf{0 . 9 8 3 1}$ \\
4 & 0 & 2.165413 & $\mathbf{0 . 9 2 9 0}$ & 0.006474 & 4.212125 & $\mathbf{0 . 9 7 7 2}$ \\
5 & 0 & 2.103061 & $\mathbf{0 . 9 2 6 6}$ & 0.007906 & 3.486211 & $\mathbf{0 . 9 7 1 8}$ \\
6 & 0 & 2.056731 & $\mathbf{0 . 9 2 8 4}$ & 0.008951 & 2.932608 & $\mathbf{0 . 9 6 5 1}$ \\
7 & 0 & 2.039132 & $\mathbf{0 . 9 3 4 4}$ & 0.00997 & 2.764115 & $\mathbf{0 . 9 6 5 6}$ \\
8 & 0 & 2.028127 & $\mathbf{0 . 9 3 8 4}$ & 0.010778 & 2.582339 & $\mathbf{0 . 7 5 0 9}$ \\
9 & 0 & 2.016057 & $\mathbf{0 . 9 4 0 0}$ & 0.012076 & 2.412671 & $\mathbf{0 . 9 6 1 8}$ \\
10 & 0 & 1.416339 & $\mathbf{0 . 9 4 1 4}$ & 0.012758 & 2.241706 & $\mathbf{0 . 9 5 5 8}$ \\
\hline
\end{tabular}

Table 12: Coverage Probabilities of Median-chart

\begin{tabular}{|l|c|c|c|l|l|c|}
\hline & \multicolumn{3}{|c|}{ Shewhart limits } & \multicolumn{3}{c|}{ Percentile limits } \\
\hline $\mathrm{n}$ & $\bar{m}-A_{7} \bar{R}$ & $\bar{m}+A_{7} \bar{R}$ & $\begin{array}{c}\text { Coverage } \\
\text { probability }\end{array}$ & $A_{7 p}^{*} \times \bar{m}$ & $A_{7 p}^{* *} \times \bar{m}$ & $\begin{array}{c}\text { Coverage } \\
\text { probability }\end{array}$ \\
\hline 2 & 0 & 2.672798 & $\mathbf{0 . 9 4 0 2}$ & 0.017322 & 25.94167 & $\mathbf{0 . 9 7 9 7}$ \\
3 & 0 & 1.964569 & $\mathbf{0 . 9 8 3 9}$ & 0.005050 & 2.61798 & $\mathbf{0 . 9 8 9 8}$ \\
4 & 0 & 1.860031 & $\mathbf{0 . 9 8 5 7}$ & 0.006207 & 2.021397 & $\mathbf{0 . 9 8 8 2}$ \\
5 & 0 & 1.793225 & $\mathbf{0 . 9 9 5 5}$ & 0.003706 & 0.870356 & $\mathbf{0 . 9 8 2 9}$ \\
6 & 0 & 1.821971 & $\mathbf{0 . 9 9 8 2}$ & 0.004146 & 0.555331 & $\mathbf{0 . 9 7 1 4}$ \\
7 & 0 & 1.833368 & $\mathbf{0 . 9 9 9 4}$ & 0.003395 & 0.334203 & $\mathbf{0 . 9 7 6 8}$ \\
8 & 0 & 1.867169 & $\mathbf{0 . 9 9 9 7}$ & 0.003796 & 0.288032 & $\mathbf{0 . 9 6 5 7}$ \\
9 & 0 & 1.893424 & $\mathbf{1 . 0 0 0 0}$ & 0.003500 & 0.209200 & $\mathbf{0 . 9 7 5 7}$ \\
10 & 0 & 1.943247 & $\mathbf{0 . 9 9 9 9}$ & 0.003954 & 0.204838 & $\mathbf{0 . 9 7 5 3}$ \\
\hline
\end{tabular}

Table 13: Coverage Probabilities of Midrange-chart

\begin{tabular}{|l|c|c|c|l|l|c|}
\hline & \multicolumn{3}{|c|}{ Shewhart limits } & \multicolumn{3}{c|}{ Percentile limits } \\
\hline $\mathrm{n}$ & $\bar{M}-A_{4} \bar{R}$ & $\bar{M}+A_{4} \bar{R}$ & $\begin{array}{c}\text { Coverage } \\
\text { probability }\end{array}$ & $A_{4 p}^{*} \times \bar{M}$ & $A_{4 p}^{* *} \times \bar{M}$ & $\begin{array}{c}\text { Coverage } \\
\text { probability }\end{array}$ \\
\hline 2 & 0 & 3.044678 & $\mathbf{1 . 0 0 0 0}$ & 0.001586 & 527.717 & $\mathbf{1 . 0 0 0 0}$ \\
3 & 0 & 2.98237 & $\mathbf{0 . 9 6 6 6}$ & 0.002362 & 65.31232 & $\mathbf{1 . 0 0 0 0}$ \\
4 & 0 & 2.819352 & $\mathbf{0 . 9 7 5 0}$ & 0.003026 & 20.49321 & $\mathbf{1 . 0 0 0 0}$ \\
5 & 0 & 3.104208 & $\mathbf{0 . 9 6 0 0}$ & 0.004535 & 16.3757 & $\mathbf{1 . 0 0 0 0}$ \\
6 & 0 & 3.152125 & $\mathbf{0 . 9 3 3 3}$ & 0.007175 & 12.59221 & $\mathbf{1 . 0 0 0 0}$ \\
7 & 0 & 3.432268 & $\mathbf{0 . 9 4 2 9}$ & 0.009641 & 10.79384 & $\mathbf{1 . 0 0 0 0}$ \\
8 & 0.231055 & 3.524695 & $\mathbf{0 . 1 1 2 5}$ & 0.011079 & 10.16944 & $\mathbf{0 . 9 6 2 5}$ \\
9 & 0.344156 & 3.768274 & $\mathbf{0 . 1 3 4 0}$ & 0.013571 & 8.64001 & $\mathbf{0 . 9 5 5 9}$ \\
10 & 0.589542 & 3.881828 & $\mathbf{0 . 0 8 0 0}$ & 0.015426 & 6.516575 & $\mathbf{0 . 8 8 0 0}$ \\
\hline
\end{tabular}


Table 14: Coverage Probabilities of Range-chart

\begin{tabular}{|l|c|c|c|c|c|c|}
\hline & \multicolumn{3}{|c|}{ Shewhart limits } & \multicolumn{3}{c|}{ Percentile limits } \\
\hline $\mathrm{n}$ & $D_{3} \bar{R}$ & $D_{4} \bar{R}$ & $\begin{array}{c}\text { Coverage } \\
\text { probability }\end{array}$ & $D_{3 p}^{*} \times \bar{R}$ & $D_{4 p}^{* *} \times \bar{R}$ & $\begin{array}{c}\text { Coverage } \\
\text { probability }\end{array}$ \\
\hline 2 & 0 & 3.542081 & $\mathbf{0 . 9 1 7 7}$ & 0 & 1063.003 & $\mathbf{1 . 0 0 0 0}$ \\
3 & 0 & 4.286345 & $\mathbf{0 . 8 9 2 3}$ & 0.000832 & 227.1966 & $\mathbf{0 . 9 9 9 8}$ \\
4 & 0 & 4.791515 & $\mathbf{0 . 8 7 7 6}$ & 0.00189 & 38.84277 & $\mathbf{0 . 9 9 7 8}$ \\
5 & 0 & 5.371677 & $\mathbf{0 . 8 6 6 7}$ & 0.004318 & 32.07793 & $\mathbf{0 . 9 9 6 0}$ \\
6 & 0 & 5.908994 & $\mathbf{0 . 8 5 6 8}$ & 0.01091 & 25.74835 & $\mathbf{0 . 9 9 2 0}$ \\
7 & 0.253946 & 6.428854 & $\mathbf{0 . 6 2 6 3}$ & 0.016039 & 20.61376 & $\mathbf{0 . 9 8 0 9}$ \\
8 & 0.505566 & 6.929234 & $\mathbf{0 . 5 9 1 1}$ & 0.01933 & 19.63494 & $\mathbf{0 . 9 7 7 0}$ \\
9 & 0.750039 & 7.402561 & $\mathbf{0 . 5 6 7 2}$ & 0.022012 & 17.75066 & $\mathbf{0 . 9 6 4 9}$ \\
10 & 0.989451 & 7.884549 & $\mathbf{0 . 5 5 0 4}$ & 0.028841 & 13.84965 & $\mathbf{0 . 9 3 4 5}$ \\
\hline
\end{tabular}

Table 15: Coverage Probabilities of SD-chart

\begin{tabular}{|l|c|c|c|c|c|c|}
\hline & \multicolumn{3}{|c|}{ Shewhart limits } & \multicolumn{3}{c|}{ Percentile limits } \\
\hline $\mathrm{n}$ & $B_{3} \bar{S}$ & $B_{4} \bar{S}$ & $\begin{array}{c}\text { Coverage } \\
\text { probability }\end{array}$ & $\boldsymbol{B}_{3 p}^{*} \times \bar{S}$ & $B_{4 p}^{* *} \times \bar{S}$ & $\begin{array}{l}\text { Coverage } \\
\text { probability }\end{array}$ \\
\hline 2 & 0 & 1.771034 & $\mathbf{0 . 9 1 7 7}$ & 0 & 2.336334 & $\mathbf{0 . 9 3 8 6}$ \\
3 & 0 & 1.962165 & $\mathbf{0 . 8 9 2 3}$ & 0.000229225 & 4.090518 & $\mathbf{0 . 9 5 7 7}$ \\
4 & 0 & 2.013366 & $\mathbf{0 . 8 4 6 9}$ & 0.000710809 & 4.368364 & $\mathbf{0 . 9 5 5 3}$ \\
5 & 0 & 2.090730 & $\mathbf{0 . 8 6 3 6}$ & 0.001701408 & 4.600906 & $\mathbf{0 . 9 5 4 8}$ \\
6 & 0.03267 & 2.145344 & $\mathbf{0 . 7 5 7 2}$ & 0.002940319 & 4.779107 & $\mathbf{0 . 9 5 5 5}$ \\
7 & 0.137953 & 2.200231 & $\mathbf{0 . 5 6 3 2}$ & 0.003507276 & 4.823440 & $\mathbf{0 . 9 5 5 4}$ \\
8 & 0.229226 & 2.248894 & $\mathbf{0 . 5 3 5 9}$ & 0.004212804 & 4.997501 & $\mathbf{0 . 9 5 6 6}$ \\
9 & 0.310508 & 2.287884 & $\mathbf{0 . 5 1 1 3}$ & 0.005456623 & 5.214973 & $\mathbf{0 . 9 5 9 6}$ \\
10 & 0.386105 & 2.332943 & $\mathbf{0 . 4 9 2 5}$ & 0.00734143 & 5.487719 & $\mathbf{0 . 9 6 1 5}$ \\
\hline
\end{tabular}

\section{Summary \& Conclusions}

The Tables 11, 12, 13, 14 and 15 show that for a true NWPD if the Shewhart limits are used in a mechanical way it would result in less confidence coefficient about the decision of process variation for mean, median, midrange, range and standard deviation charts. Hence if a data is confirmed to follow NWPD, the usage of Shewhart constants in all the above charts is not advisable and exclusive evaluation and application of NWPD constants is preferable in statistical quality control.

\section{Acknowledgments}

The authors thank the Editor and the reviewers for their helpful suggestions, comments and encouragement, which helped in improving the final version of the paper.

\section{References}

1. Betul kan and Berna Yaziki The Individual Control Charts for BURR distributed data In proceedings of the ninth WSEAS International Conference on Applied Mathematics, Istanbul, Trukey, pages 645-649, 2006. 
2. Chan. Lai. K., and Heng. J. Cui Skewness Correction $\bar{x}$ and R-charts for skewed distributions Naval Research Logistics, 50:1-19, 2003.

3. Edgeman. R.L Inverse Gaussian control charts Australian Journal of Statistics, 31(1):435-446, 1989

4. Gonzalez. I., and Viles. I., Semi-Economic design of Mean control charts assuming Gamma Distribution Economic Quality Control, 15:109-118, 2000.

5. Kantam. R.R.L., and Sriram. B., Variable control charts based on Gamma distribution IAPQR Transactions, 26(2): 63-67, 2001.

6. Kantam R.R.L., Vasudeva Rao. A., and Srinivasa Rao. G., Control Charts for Log-logistic Distribution Economic Quality Control, 21(1):77-86, 2006.

7. Kantam. R.R.L., and Srinivasa Rao. B., An improved dispersion control charts for process variate International journal of Mathematics and Applied Statistics, 1(1): 19-24, 2010.

8. Nasiru. S., and Luguterah. A., The new Weibull-Pareto distribution Pakistan Journal of Statistics and Operations Research, 11(1):103-114, 2015.

9. Srinivasa Rao B., and Sarath babu. G., Variable control Charts based on Linear Failure Rate Model International Joural of Statistics and Systems, 7(3):331-341, 2012.

10. Srinivasa Rao B., and Kantam. R.R.L., Mean and range charts for skewed distributions - A comparison based on half logistic distribution Pakistan Journal of Statistics, 28(4):437-444, 2012.

11. Subba Rao. R., and Kantam. R.R.L., Variable control charts for process mean with reference to double exponential distribution Acta Cinica Indica, 34(4): 1925-1930, 2008. 